\title{
El universalismo crítico de Leopoldo Zea. Una epistemología dialéctica para la historia de las ideas y la filosofía de la historia
}

\section{Leopoldo Zea's critical universalism. A dialectical epistemology for the history of ideas and philosophy of history}

Iver A. Beltrán-García (iivehr@hotmail.com) Universidad de Chalcatongo (Oaxaca, México) https://orcid.org/0000-0001-9761-9878

\begin{abstract}
Clarifying a set of epistemological and axiological stances and ideas of Zea (historicist realitivism, circumstantialism, perspectivism, pragmatism, political approach, rational autonomy of the theory, idea of concrete universality, dependency problem, dialectical assimilation), this paper elaborates the concept of critical universalism, which tie them up in an organized unit, and develops the structure and dynamism of this concept, describing its dialectic. The methodology is interpretative in the sense of a conceptual elaboration of the explicit and implicit contents in the work of this philosopher. Through the concept of critical universalism and its dialectics, the complementary relationship between the historicist and pragmatic-political criticism of the Mexican philosopher and his quest for universal knowledge and values are made visible, and a method is provided to study the life of knowledge and values in its concrete historicity and its political implications, without renouncing rational universality; method that, beyond the work of Zea, can be useful for the history of ideas and the philosophy of history in general.
\end{abstract}

Key words: historicism, pragmatism, engagement, universalism, dialectic.

\section{Resumen}

El artículo toma como base un conjunto de ideas y posturas epistemológicas y axiológicas de Zea (relativismo historicista, circunstancialismo, perspectivismo, pragmatismo, enfoque político, autonomía racional de la teoría, idea de la universalidad concreta, problema de la dependencia, asimilación dialéctica), las clarifica y precisa, elabora el concepto de universalismo crítico, que las articula en una unidad organizada, y desarrolla la estructura y el dinamismo de ese concepto describiendo su dialéctica. La metodología es interpretativa en el sentido de una elaboración conceptual de los contenidos explícitos e implícitos en la obra de este filósofo. A través del concepto de universalismo crítico y su dialéctica, se hace visible la relación complementaria entre la crítica historicista y pragmático-política del filósofo mexicano y su afán de conocimientos y valores universales, y se proporciona un método para estudiar la vida de los conocimientos y valores en su 
historicidad concreta y en sus implicaciones políticas, sin por ello renunciar a la universalidad racional; método que, más allá de la obra de Zea, puede ser útil para la historia de las ideas y la filosofía de la historia en general.

Palabras clave: historicismo, pragmatismo, compromiso, universalismo, dialéctica.

\section{Introducción}

El objetivo del presente artículo es explicitar y elaborar conceptualmente la estructura y unidad de un conjunto de posturas e ideas epistemológicas y axiológicas que están a la base del trabajo filosófico de Leopoldo Zea, así como su dinamismo y utilidad en general para la historia de las ideas y para la filosofía de la historia, a través del concepto de universalismo crítico y en la descripción de la dialéctica del universalismo crítico. Las posturas son el relativismo historicista, el circunstancialismo, el perspectivismo y el enfoque político y, las ideas, la autonomía racional de la teoría, la idea de la universalidad concreta, el problema de la dependencia y la propuesta de asimilación dialéctica del pasado histórico.

El método del artículo es interpretativo, no en el sentido de conectar las ideas y posturas con su contexto teórico o histórico, sino en el de operar sobre ellas un proceso de elaboración conceptual. Tal elaboración se propone, por una parte, hacer explícitos determinados contenidos de los textos de este filósofo; pero también desarrollar y complementar esos contenidos hasta ir más allá de ellos. Así, aunque el concepto de universalismo crítico añade una elaboración a las posturas e ideas del filósofo mexicano, aún permanece en el ámbito de su pensamiento; en cambio, la dialéctica del universalismo crítico, aunque se basa en las mismas y sirve para esclarecerlas, ya incluye estructuras y conceptos que no pueden ser atribuidos a su pensamiento. Este proceder metodológico se justifica en la medida que establece claramente los límites entre los aportes explícitos e implícitos del autor interpretado y los del intérprete; pero también en cuanto la historia de las ideas, además de aspirar a un conocimiento riguroso, se concibe como un diálogo con el pasado.

La importancia del artículo es doble. Por un lado, y principalmente a través del concepto de universalismo crítico, permite profundizar la comprensión de las ideas y posturas epistemológicas y axiológicas básicas en la obra de Zea. Por otro lado, la dialéctica del universalismo crítico, además de que contribuye a tal profundización, amplía y complementa dichas ideas a partir de su propio espíritu, aportando con ello un método que puede ser útil a la historia de las ideas y la filosofía de la historia en general.

Tres aclaraciones. Primero: una parte de la elaboración conceptual, específicamente la de los principios y las bases, será comunicada por extenso en otro lugar, y por ello en la primera sección me limito a hacer de ella una exposición sintética. Segundo: debido a las normas editoriales, en la bibliografía únicamente registro las fuentes citadas a lo largo del artículo, pero debe entenderse que la investigación ha sido efectuada sobre una base bibliográfica más amplia. Tercero: las cursivas y mayúsculas, así como los encabezados entre corchetes, son únicamente un recurso expositivo de coherencia y énfasis. 


\section{Base epistemológica y política}

En esta sección explico los principios de la base epistemológica y de la base política, y articulo ambas bases, para poder elaborar en la siguiente sección el concepto de universalismo crítico y describir la dialéctica del universalismo crítico.

La base epistemológica en el pensamiento de este filósofo está conformada por los siguientes principios:

[Base epistemológica]

(1) Principios críticos

(a) Relativismo historicista, moderado, de carácter epistemológico y con dimensión axiológica. Comprende: (i) un Circunstancialismo y (ii) un Perspectivismo.

(b) Pragmatismo, moderado, de carácter epistemológico y con dimensión axiológica.

(c) Enfoque político de los conocimientos y los valores

(2) Principios constructivos

(a) Autonomía racional de la teoría

(b) Idea de la universalidad concreta

Para explicar estos principios, conviene efectuar previamente tres precisiones terminológicas y conceptuales:

\section{[Precisiones]}

Zea aplica las ideas y posturas estudiadas, las cuales tienen carácter epistemológico, a conocimientos y valores por igual; por tanto, trata a los valores como realidades que pueden ser conocidas. Sin embargo, dado que no nos ha dejado un análisis sobre la materia, y puesto que desarrollar ese aspecto de su pensamiento implica plantear tesis exegéticas cuya elaboración requiere un lugar específico, aquí únicamente indico la problemática, aludiendo a ella cuando atribuyo al relativismo historicista y al pragmatismo de este filósofo un carácter epistemológico "con dimensión axiológica".

Dado que el conocer y el valorar en general pueden entenderse como actividad o como producto, en lo que sigue denominaré "teoría" o "actividad teórica" a la actividad cognoscitiva (científica y no científica) y de valoración, y reservaré el nombre de "conocimiento" o "valor" para los productos. Los términos "conocer" y "valorar", por su parte, quedan reservados para la unidad entre la actividad y los productos. Así, la actividad teórica, o teoría, tiene dos dimensiones, la epistemológica y la axiológica.

Para coincidir con el uso que el filósofo mexicano hace del término y sus derivados, en este lugar entiendo la "concreción" no como una mayor determinación entre conceptos, sino como determinación por la existencia histórica. Así, en vez de considerar a unos conceptos más concretos que a otros (como el de pintor respecto al de artista), englobo a todos los conceptos como objetos 
abstractos, y a los seres que dan existencia histórica a esos conceptos (por ejemplo, el individuo humano históricamente existente que es pintor o músico) como objetos concretos.

Pasemos, pues, a la explicación de los principios de la Base epistemológica.

Relativismo historicista. Zea niega que existan "verdades eternas", es decir, conocimientos o valores que sean eso, conocimientos o valores y no otra cosa, al margen de toda circunstancia histórica. Los conocimientos y valores de una circunstancia histórica no lo son en otra, en tanto que esa otra circunstancia difiere de la primera; pero pueden serlo por cuanto una y otra circunstancia presentan semejanzas. Los conocimientos y valores de distintas circunstancias se complementan, no se excluyen entre sí (es decir, no se contradicen o invalidan).

Circunstancialismo. Cuando enlaza el conocimiento o el valor con la circunstancia histórica, este filósofo no reduce la historia a una atomización desarticulada de circunstancias abstractas. En primer lugar, para el filósofo mexicano la circunstancia es todo aquello con lo que el hombre se relaciona, sea en sentido conservador, sea en sentido transformador (idea compleja, pues el hombre puede relacionarse con su propio ser). En segundo lugar, Zea concibe a la circunstancia como una red de semejanzas y diferencias con las otras circunstancias, de manera que, así como no hay dos circunstancias totalmente idénticas, tampoco las hay totalmente distintas. Esto implica, en tercer lugar, que hay circunstancias exclusivas del individuo, pero también compartidas por un grupo o incluso por todos los seres humanos. Y, por último, para este filósofo la circunstancia no es solo el vacío enlace abstracto de tiempo y espacio, sino un plexo de determinaciones históricas concretas, entre ellas las económicas, políticas, sociales y culturales.

Perspectivismo. El conocimiento y el valor, a partir de este relativismo historicista y circunstancialismo, aparecen en el pensamiento del filósofo mexicano como perspectivas. Teniendo la teoría necesariamente, además de la realidad como principio unificador, a la circunstancia histórica como principio diversificador, el sujeto no conoce o valora el todo de la realidad, sino que, inevitablemente, solo puede conocer o valorar de ella un aspecto en cada circunstancia. Este perspectivismo trae dos consecuencias. Por una parte, hay perspectivas (conocimientos, valores) para las circunstancias individuales, pero también para las de los grupos sociales y para las que se extienden a la humanidad en su conjunto. En segundo lugar, las perspectivas individuales o de grupo, si bien carecen de universalidad, pueden superar su particularidad al ser comunicadas e integradas en visiones más amplias de la realidad, pues el aspecto de la realidad que capta una perspectiva no excluye el que aprehende otra.

Pragmatismo. En la circunstancia histórica en general a la que vincula todo conocimiento y valor, Zea destaca el aspecto de la práctica, entendida como actividad humana en general. Aunque este filósofo se limita a oponer la teoría a la práctica, es necesario efectuar una distinción más para la mejor comprensión de sus planteamientos; distinción que ha de entenderse como herramienta interpretativa y no como aporte del autor interpretado. La práctica, como actividad humana en general, se bifurca en una práctica teórica y una práctica no teórica. La práctica teórica, o simplemente teoría, es la que tiene como objetivo principal la generación y transmisión del conocimiento, a diferencia de otras formas de práctica, como la económica o la política, las cuales, si bien utilizan conocimiento, tienen algo diferente como fin principal. La práctica no teórica es no teórica no porque no incorpore conocimiento, sino porque el conocimiento no constituye su fin 
principal. La base epistemológica del filósofo mexicano, a partir de mi distinción, puede calificarse como pragmática en el sentido de que la teoría está subordinada a la práctica no teórica, puesto que el fin último de la teoría, visto en una perspectiva más amplia, solo constituye un fin intermedio de alguna práctica no teórica, como la economía o la política, la religión o el arte.

Enfoque político. Pero Zea, así como destaca el aspecto de la práctica entre los otros aspectos de la circunstancia histórica, también pone de relieve una cierta práctica entre las distintas formas de práctica no teórica: la práctica política. Es decir, en su historia de las ideas y en su filosofía de la historia, si bien es cierto que explica los conocimientos y los valores relacionándolos con la circunstancia histórica en general, da prioridad al aspecto práctico de cada circunstancia, y dentro del aspecto práctico enfatiza la actividad política en su sentido no teórico. De esta manera, conocimientos y valores suelen ser explicados, en última instancia, en su articulación a proyectos políticos de dominación o de liberación. Como veremos, el sentido de lo político en el pensamiento de Zea se aclara y especifica a través de su base política.

El carácter moderado del relativismo historicista y del pragmatismo de este filósofo se debe a que ambos dan lugar a la idea de la universalidad concreta y a la autonomía racional de la teoría.

Idea de la universalidad concreta. El relativismo es radical si no admite la posibilidad de los conocimientos o valores universales, y moderado si la admite. Universal no significa aquí lo mismo que absoluto. Para el relativismo un conocimiento o valor no puede ser absoluto (independiente de todo contexto), pero sí puede ser universal (ser conocimiento o valor para todos los sujetos cognoscentes de un determinado contexto). En el caso del filósofo mexicano, el relativismo historicista es compatible con la idea de que el conocimiento o el valor puede tener universalidad, pero no una universalidad abstracta, conforme a la cual el conocimiento o el valor es lo que es, conocimiento o valor, para todos los sujetos cognoscentes o de valoración posibles al margen de su contexto contingente, sino una universalidad concreta, de acuerdo con la cual el conocimiento o el valor es lo que es, conocimiento o valor, para todos los sujetos existentes dentro de un contexto determinado, siendo el contexto la circunstancia histórica en el caso de Zea.

Autonomía racional de la teoría. El pragmatismo es radical si excluye la posibilidad de autonomía racional en la práctica teórica, y moderado si la incluye. Esta autonomía se refiere a que la teoría posee: unos principios lógicos, que rigen la relación entre las ideas; unos principios epistemológicos, fundamento de la relación entre las ideas y la realidad en tanto esas ideas tratan de captar tal realidad; y unos principios axiológicos, relativos a la relación entre el ser y el valor, y a la relación entre el conocer y el valorar. Si la teoría es racionalmente autónoma, sus principios no son los de la práctica no teórica a la que se subordina, sino los principios lógicos, epistemológicos y axiológicos que, aunque evolucionando históricamente, tienen como sentido garantizar el cumplimiento del fin que es propio a la misma teoría: el conocimiento o el valor. Por tanto, la subordinación de la teoría a la actividad no teórica no implica una identificación de la teoría con una racionalidad limitadamente instrumental, pues, en uso de su autonomía racional, la teoría puede llegar a desenvolverse como racionalidad de fines y de principios.

Tales son los principios de la base epistemológica en el pensamiento de este filósofo. Además de explicar sintéticamente cuáles son y en qué consisten, el tema del presente artículo exige hacer visible en el pensamiento del filósofo mexicano la relación entre el enfoque político y la base política. 
No perdamos de vista que el enfoque político es un principio de la base epistemológica, mientras que la base política se refiere a un conjunto de principios diferente a los de la base epistemológica.

Los principios que conforman la base política son los siguientes:

[Base política]

(1) El problema de la dependencia

(2) La asimilación dialéctica del pasado histórico

El problema de la dependencia se refiere a la dominación de unos hombres sobre otros al interior de una colectividad o entre colectividades (por ejemplo, entre comunidades o sociedades, entre grupos sociales o países, entre naciones o regiones). Tiene múltiples dimensiones, como las económicas, políticas, sociales y culturales; y se plantea en el ámbito de la práctica no teórica, es decir, se refiere a una situación real y a la necesidad de su transformación, aunque incorpora la práctica teórica entre sus componentes.

La asimilación dialéctica consiste en una actividad teórica de conocimiento del pasado histórico, de la realidad presente, natural y social, y del propio ser. Es dialéctica porque, (i) ante el rechazo del pasado histórico y la realidad presente y la consecuente negación del propio ser, de la misma manera que (ii) ante la contrastante afirmación de los mismos (pasado, realidad, ser) que soslaya u oculta los problemas que ellos llevan consigo y acumulan sin resolver, (iii) la asimilación toma conciencia tanto de los aspectos negativos, destacadamente las estructuras de dominación, como de todo aquello que forma parte del propio ser, de todo lo que no podemos negar sin negarnos a nosotros mismos y que contribuye a liberarnos de esas estructuras.

La asimilación dialéctica se plantea como solución al problema de la dependencia, pero una solución parcial. Esto se debe al carácter teórico de aquella, mientras que este problema pertenece al ámbito de la práctica no teórica. Así, la asimilación dialéctica, al contribuir a la solución del problema de la dependencia, debe articularse y subordinarse a una práctica no teórica de liberación que la integra y a la vez la trasciende.

Esta base política, con los principios y las relaciones explicados, se relacionan con el enfoque político como principio de la base epistemológica, en el sentido de que aquella base especifica o precisa este enfoque.

Quedan así explicadas la base epistemológica y la base política a través de sus principios críticos y constructivos, además del vínculo entre ambas bases.

\section{El universalismo crítico y su dialéctica}

A partir de lo explicado, y utilizándolo como materia prima, en esta sección elaboro el concepto de universalismo crítico, describo la dialéctica del universalismo crítico y bosquejo el diálogo a que el concepto y la descripción dan pie con la bibliografía secundaria. 
El universalismo crítico de Zea, que articula su base epistemológica y su base política, puede ser formulado de la siguiente forma:

\section{[Universalismo crítico]}

(a) Postura epistemológica con dimensión axiológica, la cual

(b) acepta la autonomía racional de la teoría respecto a la actividad no teórica, y la posibilidad de conocimientos y valores universales, pero que

(c) entiende esa universalidad en un sentido concreto,

(d) haciéndola depender de la circunstancia histórica y

(e) subordinando toda teoría a la práctica no teórica (especialmente a la política).

El universalismo crítico, como concepto, no es elaborado por este filósofo ni aparece en sus escritos o en la bibliografía secundaria, pero hace explícitas las conexiones y la unidad organizada que en esos escritos tienen entre sí efectivamente las ideas y posturas explicadas en la sección anterior. Se trata, pues, de un concepto interpretativo complejo.

La dialéctica del universalismo crítico, que presupone el concepto así elaborado, es expuesta en seguida a través de una descripción interna, una descripción externa y unas observaciones.

[Dialéctica del universalismo crítico. Descripción interna: los momentos]

Primer momento. En su historia de las ideas y en su filosofía de la historia, este filósofo toma como punto de partida los conocimientos o valores con pretensión de universalidad en la cultura occidental. La universalidad de esos conocimientos y valores consiste en que son válidos para todos los sujetos cognoscentes o de valoración posibles; y su validez, en ser conocimientos o valores respectivamente, y no otra cosa. Las determinaciones que marcan este momento son la totalidad y la posibilidad de los sujetos: la validez se refiere a la totalidad de los sujetos, no a un conjunto de ellos en particular, y a los sujetos posibles, no a los existentes.

Segundo momento. El filósofo mexicano pone en acción su crítica, es decir, su relativismo historicista, su circunstancialismo, su perspectivismo, su pragmatismo y su enfoque político. A través de esta crítica muestra que los conocimientos y valores que pretendían ser válidos para todos los sujetos cognoscentes y de valoración posibles, únicamente lo son para algunos sujetos existentes, en una determinada circunstancia histórica, y que, por tanto, constituyen perspectivas parciales y limitadas. Las nuevas determinaciones de los conocimientos y valores son la particularidad y la concreción: los conocimientos y valores no son universales porque solo lo son para algunos sujetos particulares y porque esos sujetos son existentes, concretos y no solo posibles.

Tercer momento. Zea insiste una y otra vez a lo largo de su obra en que lo particular y concreto, lejos de ser el destino de la filosofía, son su punto de partida en el camino de la universalidad. Los conceptos clave en esta búsqueda de conocimientos y valores universales a partir de la particularidad y la concreción, son los de circunstancia y perspectiva: hay circunstancias compartidas por todos los seres humanos, y esas circunstancias dan base a perspectivas igualmente compartidas, es decir, a conocimientos y valores universales. Pero esta universalidad no es la misma que en el primer momento, la supera dialécticamente: conserva la determinación de la totalidad de 
ese primer momento, pero a la vez la determinación de concreción del segundo momento; o, dicho negativamente: cancela del primer momento la posibilidad y del segundo momento la particularidad. La universalidad concreta se refiere a que los conocimientos y valores son válidos para todos los sujetos existentes dentro de una determinada circunstancia histórica.

[Dialéctica del universalismo crítico. Descripción externa: los ciclos]

En el tercer momento hay una especie de vuelta al primer momento, pero una vuelta en espiral, un enriquecimiento de sentido, ya que la universalidad es pensada como concreta y ya no como abstracta. Ese enriquecimiento implica un nivel superior de conciencia, pues guarda como memoria la experiencia del primer momento y del segundo momento.

La vuelta en espiral del tercer momento al primer momento implica que un ciclo se cierra y otro se abre. El que se cierra es el de la crítica (segundo momento) a la universalidad abstracta (primer momento) y de superación de la oposición (tercer momento). El que se abre comienza en el mismo tercer momento que cierra al ciclo anterior; ese tercer momento, por tanto, puede ser visto desde el primer ciclo como momento de cierre, o desde el siguiente ciclo como momento de apertura, como un nuevo primer momento.

Unos sujetos concretos que, en el primer ciclo, como parte de un proceso de liberación, ejercieron la crítica historicista, pragmática y política sobre la universalidad abstracta, pueden en el siguiente ciclo transformarse en (o ser sustituidos por) los sujetos de una estructura de dominación, y utilizar la universalidad concreta como legitimación ideológica del estado de cosas presentándola como universalidad abstracta. Esta posibilidad de que el universal concreto se transforme en una nueva universalidad abstracta, es lo que hace necesaria una nueva crítica historicista, pragmática, política, $y$, en su caso, una nueva superación dialéctica o universalidad concreta: el segundo momento y tercer momento del segundo ciclo.

La dialéctica del universalismo crítico es una dialéctica abierta. Esto quiere decir que no tiene un inicio y un término absolutos. Lo que he considerado como primer ciclo puede en realidad ser entendido como la continuación de ciclos anteriores. De la misma manera, cualquier ciclo puede ser continuado por otros.

[Observaciones: estatuto epistemológico]

La dialéctica del universalismo crítico no es una estructura objetiva en el devenir histórico de los conocimientos y los valores. Se trata, en cambio, de un método teórico para someterlos a examen historicista y pragmático desde un enfoque político, sin renunciar a la autonomía racional de la teoría y a la universalidad de estos.

La dialéctica del universalismo crítico, como método teórico, puede ser utilizado como herramienta principal, o coordinada o subordinada, en la historia de las ideas y en la filosofía de la historia. Sin embargo, y por supuesto, no exige retomar los mismos autores y las mismas corrientes filosóficas que Zea hizo suyas, sino que está abierto a la adopción y adaptación de corrientes actuales. Considérense, por ejemplo, las herramientas que pueden proporcionar el pensamiento 
postmoderno y el neopragmatismo al momento crítico, y las de la teoría crítica o la hermenéutica analógica para la construcción de una universalidad concreta.

En la dialéctica del universalismo crítico, la descripción interna es implícita, pero como se verá en la siguiente sección, también resulta necesaria para explicar la obra en general de este filósofo. En cambio, su descripción externa debe más al lector de su obra que al mismo filósofo mexicano; y, no obstante, esta segunda descripción, además de ser coherente con la primera, es exigida por su espíritu político y liberador, pues, si toda universalidad puede transformarse en universalidad abstracta y herramienta de dominación (incluso la universalidad concreta producida a través de la crítica historicista y pragmática), entonces esa crítica debe renovarse una y otra vez y hacerse permanente.

Tal es, pues, la dialéctica del universalismo crítico.

El concepto de universalismo crítico y su dialéctica, aunque en este lugar se expresan abstractamente, son sin embargo producto de un diálogo con la abundante bibliografía secundaria sobre Zea. Aquí me limito a indicar los puntos básicos de ese diálogo y algunos de los interlocutores.

Cientificidad. Hay señalamientos relativos a la cientificidad y objetividad de este filósofo a causa de su relativismo historicista, su pragmatismo y su enfoque político. Raat ve en Zea una rebelión contra el pensamiento científico y la lógica inductiva, "un alejamiento de lo externo y un acercamiento a lo interno, de lo objetivo a lo subjetivo, de lo universal a lo particular, de la historia científica a la historia como arte romántico o como filosofía" (Raat 1970:180). Hale distingue una "renuncia a la objetividad como historiador" (Hale 1970:301). Davis se pregunta: "¿excluye la visión idealista y neohegeliana de Zea una aproximación más empírica o sicológica behaviorista en la búsqueda de la dirección del proceso histórico?" (Davis 1972:45). Kourím, respecto a Dialéctica de la conciencia americana, constatando que "los hechos históricos parecen apoyar y confirmar contundentemente el esquema dialéctico aplicado", plantea una cuestión similar: “¿no son [los hechos] algunas veces un poco comprimidos, plegados en su sentido, para colocarse mejor ahí?" (Kourím 1992:124). Y Lizcano observa que "en sus formulaciones [de Zea] no se percibe con la suficiente nitidez la necesaria deformación de la realidad provocada por los supuestos teóricos que también necesariamente tiene el conocedor de la misma" (Lizcano 2004:234). Zea responde a los tres primeros operando sobre ellos su relativismo historicista, su pragmatismo y su enfoque político, y resalta que, en un mundo marcado por dominación de unos hombres sobre otros, la petición de objetividad y cientificidad enmascara el intento de despolitizar al intelectual, de no permitirle "ningún compromiso con la realidad que, como hombre concreto, le es propia" (Zea 1974:15); pero con esto no confirma los señalamientos: en la dialéctica del universalismo crítico, por ejemplo, junto con un ejercicio intelectual consciente de la circunstancia histórica y del compromiso político, es claro el afán por la autonomía racional y por la universalidad de los conocimientos (e implícitamente de los valores): "Toda la filosofía de la historia, por supuesto, implica un proyecto [...] Proyecto, insistimos, que implica no atenerse a los hechos; pero que no implicó ignorarlos" (Zea 1978:25). Zea orienta su pensamiento en general a la transformación de los hechos, y esa transformación exige como condición de posibilidad el conocimiento objetivo y científico de los mismos.

Principios. Hernández, que estudia ampliamente y en detalle el historicismo, circunstancialismo, perspectivismo y pragmatismo de Zea en relación con el pensamiento de Ortega y Gasset, considera 
desde un punto de vista epistemológico que la filosofía del primero es "integralista" (Hernández 2004:300) y no relativista, en el sentido de que la semejanza entre circunstancias y la complementariedad entre perspectivas permiten la integración de los conocimientos universales. Sin embargo, el integralismo, al rechazar el posible carácter absoluto de los conocimientos y no su posible universalidad, constituye un relativismo historicista, tal como esta postura fue caracterizada en la sección anterior.

Método. Villegas escribe que "las verdades 'filosóficas' o son circunstanciales o son generales, pero no ambas cosas a la vez" (Villegas 1960:152); y Villoro, refiriéndose a Zea y la filosofía nacionalista latinoamericana del segundo tercio del siglo XX, apunta que "la manera como se alcanzaría algo universal a partir del examen de un objeto concreto nunca se precisó" (Villoro 1987:88). Ambos tienen razón en cuanto a que la base metodológica de la idea de la universalidad concreta no es ni la lógica formal ni la fenomenología; pero debe agregarse inmediatamente que eso se debe a que tal base tiene en cambio naturaleza dialéctica, y más específicamente, que corresponde a la dialéctica del universalismo crítico (al menos en su descripción interna). Aspectos específicos de esa dialéctica han sido analizados por Beltrán, que en el movimiento operado por Zea de lo concreto a lo universal ve "una dialéctica del reconocimiento" (Beltrán 2017:254), y que extrae de los textos de Zea una "dialéctica de la utopía" (Beltrán 2020:179) para comprender y enfrentar la degradación ideológica de una utopía que arriba al poder.

Universalidad concreta. El concepto de universalidad concreta, que con toda razón Medin considera la "piedra angular" (Medin 2005:40) del pensamiento de Zea, exige su desarrollo en el universalismo crítico y en la dialéctica del universalismo crítico, debido a que tiene como sentido fundamental la crítica al fenómeno de la universalidad abstracta, pero no precisa por sí mismo la estructura y el dinamismo de esa crítica. Respecto a ese sentido fundamental, Friz considera que el universalismo de Zea, al adscribir la cultura americana a la europea, es "relativamente fuerte" (Friz 2019:68) (y no fuerte en sentido absoluto) debido a que a través del concepto de universalidad concreta critica la actitud excluyente de la cultura europea hacia la diversidad cultural; y Reyes-Ruiz apunta que para Zea la mejor forma de contrarrestar los prejuicios de la cultura occidental sobre el hombre "no es apelando otra vez a una supuesta universalidad abstracta, sino a la universalidad concreta presente en la expresiones culturales de otros pueblos" (Reyes-Ruiz 2020:14).

Relación teoría/praxis. Kourím considera que Zea fracasa en su empresa de universalizar las ideas y los valores de la cultura occidental, y que ese fracaso se debe a una ruptura entre la conciencia de la realidad y la acción revolucionaria: "por una moralización de la realidad, se debe conseguir su transformación [...] Solo a través de ella [de la moralidad] (en el proceso de 'acumulación de ser') el ser americano se autentiza, logra un valor universal" (Kourím 1974:98) y Shulgovsky destaca que, para Zea, "las ideas son la fuerza principal motora o el freno (según las condiciones) del desarrollo de los países latinoamericanos" (Shulgovsky 1979:28). En contraste, la dialéctica del universalismo crítico nos muestra en el pensamiento de Zea una "relación esencial entre teoría y praxis" (Medin 1983:146): una articulación necesaria entre la teoría (las ideas estudiadas, pero también las propias ideas) y la práctica política multidimensional, es decir, económica, política y social, no únicamente cultural. Relación en la cual la teoría, aunque se subordina a la práctica no teórica, no pierde su autonomía racional, ni pretende ser el elemento central y básico en la práctica no teórica; en este sentido, "Zea logra navegar con éxito entre el Escila y el Caribdis del economicismo y el ideologismo" (Guadarrama 2013:37). 
Actitudes. Gómez-Martínez observa dos actitudes en la obra de Zea: la actitud de confrontación, que implica una toma de conciencia de la propia opresión y "una supuesta superioridad moral" (Gómez-Martínez 1997:49) y la actitud de oposición dialógica. En realidad, ambas actitudes (la primera despojada del énfasis excesivo que implica considerarla una confrontación) pueden ser pensadas como elementos estructurales en la filosofía de Zea, pues se refieren a sendos momentos de su dialéctica del universalismo crítico: el segundo momento, de crítica a la universalidad concreta, y el tercer momento, de construcción de una universalidad concreta.

Forma expresiva. Roig señala el "carácter recurrente" (Roig 1981:265) de la obra de Zea; Vera su "repertorio limitado de temas sobre el cual reflexiona una y otra vez" (Vera 1982:290); el mismo Zea "un filosofar reiterativo pero no repetitivo" (Zea 1988b:15); y López, su predilección temática, entendida como "un sucesivo enriquecimiento conceptual que, al profundizar, se propone cada vez más aproximaciones a las raíces mismas de los problemas que trata" (López 1989:45). A mi juicio, aquello a lo que se apunta en los cuatro casos, más profundamente que a la transmisión pedagógica ante diferentes audiencias y a la recontextualización de los mismos temas en circunstancias distintas, es a la dialéctica del universalismo crítico: el pensamiento de Zea retorna una y otra vez a los mismos temas, pero en sus mejores momentos con un nivel de conciencia más alto, pues, como ya queda explicado, el retorno trae consigo la memoria del proceso previo de crítica y universalización.

De esta manera, ha sido expuesto el concepto de universalismo crítico, su dialéctica y algunas líneas de diálogo originadas en ellos.

\section{Documentación}

En esta sección documento la presencia de la base epistemológica, la base política, el universalismo crítico y la dialéctica del universalismo crítico en el trabajo filosófico de Zea. Aunque el espacio no permite referir un conjunto más amplio de textos, tomo en cuenta los diversos momentos y aspectos de su obra.

Veamos primero la presencia de los principios críticos y los principios constructivos.

En El positivismo en México de 1943, obra de historia de las ideas, Zea retoma explícitamente planteamientos historicistas de Ortega y Gasset, Croce, Groethuysen, Scheler y Mannheim, y a partir de ellos, sostiene que "las verdades de la filosofía no son verdades absolutas en el sentido de eternas, sino absolutas en un sentido circunstancial, que valen en forma absoluta para una circunstancia dada" (Zea 1943:21), y que "los filosofemas, los conceptos, las verdades de la filosofía son considerados como expresiones de una cierta visión del mundo en la cual participan las demás formas de la cultura" (Zea 1943:22). Los dos últimos, Mannheim y Scheler, aportan a Zea elementos para el estudio pragmático de las ideas: "Karl Mannheim sostiene la tesis, a la que me adhiero, de que toda ideología es expresión de una determinada clase social [y] Max Scheler ha mostrado cómo una clase en el poder tiende a una filosofía de carácter estático, y una clase sin poder tiende a una filosofía de carácter dinámico" (Zea 1943:38).

En el libro En torno a una filosofía americana de 1945, importante para la filosofía de la filosofía y para la filosofía de la historia de Zea, este observa (primera parte, apartados III a V) que a partir de 
pensadores como Ortega y Gasset y Mannheim la reflexión filosófica reconoce la radical historicidad del hombre y de lo humano en tanto que cada individuo y cada grupo social tiene un punto de vista propio de acuerdo con su concepción de la vida, y la necesidad de tomar como objeto los problemas de su circunstancia y contribuir a solucionarlos. Zea también señala que la teoría debe ser fundamento de la práctica (de la no teórica, se entiende), en el sentido de que utiliza la experiencia del pasado para orientar al hombre en el presente y futuro; y que los problemas de la práctica (no teórica) a cuya solución contribuye la filosofía, son los morales y los políticos. Zea reconoce la posibilidad de un conocimiento universal, puesto que, así como hay circunstancias individuales, de grupo y universales, hay también verdades individuales, de grupo y universales: "Todos los hombres para ser hombres participan de una circunstancia que les es propia: la humanidad [...] existen verdades que pueden valer para toda la humanidad, para todos los hombres" (Zea 1945:31). Es la idea de la universalidad concreta, aunque presupuesta, aun no formulada como tal. De la misma manera, Zea supone que la teoría necesita preservar su autonomía racional para cumplir su fin de generar conocimiento, pues el error y el engaño pueden traer consigo el fracaso de la acción humana: "la teoría trata de captar, en un reducido número de símbolos, el conjunto de las experiencias humanas realizadas, para que partiendo de las mismas utilice los aciertos y evite los errores" (Zea 1945:68). Zea incorpora estas ideas de En torno a una filosofía americana en los capítulos II-IV y $\mathrm{X}$ de América como conciencia, que constituye un resumen de sus trabajos anteriores.

La idea de la universalidad concreta, implícita en los textos tempranos de Zea, solo se hace explícita a fines de los años cuarenta y comienzos de los cincuenta en sus trabajos de filosofía de lo mexicano. En "Dialéctica de la conciencia en México", conferencia de 1951 incluida en La filosofía como compromiso y otros ensayos de 1952, Zea observa que: "En lo concreto, lo más concreto como es el hombre de carne y hueso, se encierra lo universal, lo que es válido para todos los hombres de carne y hueso, para todos los semejantes" (Zea 1952a:213); en Conciencia y posibilidad del mexicano de 1952 plantea que el éxito de las teorías y doctrinas de la Revolución Mexicana "dependerá de la capacidad de sus pensadores para captar el sentido universal de sus expresiones [...] convirtiendo lo concreto circunstancial en concreto universal (Zea 1952b:18); y en el primer capítulo de El occidente y la conciencia de México de 1953, diferentes concepciones sobre el ser del hombre son interpretadas como parte de una circunstancia histórica y en articulación a una determinada práctica política, de manera que la crítica de esas concepciones origina una forma diferente de pensar al ser humano: "Lo humano se encuentra en ese modo de ser concreto que es cada hombre en particular" (Zea 1953b:14).

En el tercer capítulo de La filosofía americana como filosofía sin más de 1969, reflexión sobre el vínculo entre la filosofía y la práctica no teórica en general, encontramos otro ejemplo referente a la autonomía racional de la teoría y a la idea de la universalidad concreta. En ese lugar, destacando que desde sus primeros escritos publicados ha sostenido que la filosofía debe tomar como punto de partida los problemas de la circunstancia particular, sostiene que "lo importante es filosofar, pura y simplemente filosofar. Esto es, enfrentarse racionalmente a los problemas que nos plantea la realidad, buscando a tales problemas la solución más amplia y adecuada" (Zea 1969:59, cursivas mías). Hacer teoría racionalmente autónoma, y orientarse a conocimientos y valores con amplitud universal. 
Así ilustrados, los principios críticos y los principios constructivos pertenecen a la base epistemológica de Zea. En cuanto a su base política, aunque ya en "Esquema para una historia del pensamiento en México" de 1946 (recogido en Ensayos sobre filosofía en la historia), Zea apuntaba que "México, o más ampliamente América, ha surgido a la historia como dependencia europea" (Zea 1948:201), es en Dos etapas del pensamiento en Hispanoamérica de 1949 donde el problema de la dependencia es puesto por Zea en el centro de su trabajo de historia de las ideas: "Siempre está patente el problema de la dependencia y la independencia del hombre hispanoamericano, llustrados, liberales, conservadores, positivistas y revolucionarios, no han hecho otra cosa que expresar en diversas épocas y con diverso lenguaje el mismo y siempre latente problema" (Zea 1949:19). En su afán de librarse política y culturalmente del yugo español, el hispanoamericano niega su pasado histórico, es decir, cierra los ojos ante su propio ser y su propia realidad y deja sin resolver los problemas heredados, y de ese modo, emprende un proceso de modernización que resulta utópico por no basarse en el conocimiento de ese ser y esa realidad y que conduce a nuevas formas de coloniaje. Para enfrentar esa situación, y siguiendo a Hegel y a Ortega, Zea propone una asimilación dialéctica del pasado histórico: "negar no significa eliminar sino asimilar, esto es, conservar [...] se es plenamente, para no tener necesidad de volver a serlo" (Zea 1948:16). Superar el pasado, sí, pero reconociendo sus problemas aun vivos en el presente, y enfrentándolos a través de un proyecto orientado por la conciencia de las propias posibilidades y limitaciones.

Conforme a lo anterior, la base epistemológica y la base política, que se articulan en el concepto de universalismo crítico, son contenidos que Zea hace explícitos en un momento u otro de su obra; en cambio, la dialéctica del universalismo crítico solo puede ser reconocida a través de la mediación de un proceso de análisis. Veamos.

En el capítulo II de El pensamiento latinoamericano de 1965, Zea observa que el nacionalismo antiimperialista de los pueblos que continúan padeciendo la dominación de Occidente no debe ser visto como amenaza, sino, por el contrario, como oportunidad para la verdadera universalización de la cultura occidental. Lo que esos pueblos plantean es que los valores de esta cultura (como libertad, justicia social, democracia, y otros artísticos, literarios, filosóficos, científicos), si bien han mejorado la vida de los hombres al interior de Occidente mismo, no han regido sin embargo la relación de Occidente con el resto del mundo, pues en esta relación han servido preponderantemente para justificar la imposición de unos intereses materiales particulares y excluyentes; que, en ese sentido, la universalización de la cultura occidental ha sido falsa, y que será verdadera únicamente cuando sus valores beneficien a todos los hombres. No hay, pues, hostilidad hacia la cultura occidental; la amenaza real para Occidente es su propio exclusivismo: "espíritu exclusivista que se niega a reconocer en otros hombres o pueblos derechos que antes ha reclamado para sí" (Zea 1965:30), ya que es ese exclusivismo lo que puede privar a Occidente de su autoridad moral y su papel educativo ante el mundo.

En esta idea de la verdadera universalización de la cultura occidental opera la dialéctica del universalismo crítico: 
[Dialéctica del universalismo crítico: universalización de la cultura occidental]

Primer momento. Zea piensa los conocimientos y los valores de Occidente como "verdades eternas" o universales abstractos, es decir, en su pretensión de ser válidos para todos los sujetos cognoscentes y de valoración posibles.

Segundo momento. Este filósofo efectúa un examen de esos conocimientos y valores desde sus principios críticos: relativismo historicista, pragmatismo y enfoque político. Dichos valores no son "ideas eternas", sino que pertenecen a una determinada cultura, la occidental, en una determinada época, la moderna, y sirven de justificación a una práctica no teórica, imperialista, de carácter político.

Tercer momento. El filósofo mexicano piensa esos mismos conocimientos y valores desde sus principios constructivos: idea de la universalidad concreta y autonomía racional de la teoría. Los valores de la cultura occidental se universalizan en la medida que, en ejercicio de la razón autónoma, son reconocidos como tales, como valores y no otra cosa, por los hombres occidentales y no occidentales que comparten la circunstancia de la expansión de Occidente sobre el mundo y, por tanto, en la medida que esos valores dejan de ser utilizados como máscaras de intereses excluyentes.

Este Tercer momento, como ya quedó explicado, puede generar un nuevo ciclo, si la universalidad concreta, resultado de un proceso de liberación, se transforma en universalidad abstracta para justificar una nueva estructura de dominación. Gómez-Martínez ofrece un ejemplo de cómo dentro de la dialéctica del universalismo crítico es históricamente necesario el paso de un primer a un segundo ciclo, pues plantea que el discurso de Zea de respeto a la diferencia, secuestrado por los centros de poder, ha venido a mostrar su lado opresor, en tanto que "se trata de un 'respeto' que paradójicamente viene a perpetuar, a justificar, el statu quo" (Gómez-Martínez 2004:40). En un primer ciclo, el universal abstracto (intolerancia hacia la diferencia) es sometido a crítica historicista y pragmático-política, produciéndose así un universal concreto (respeto a la diferencia), pero ese universal concreto, enraizado en una práctica de liberación, es utilizado por una estructura de dominación, de modo que se transforma en un nuevo universal abstracto, el cual exige otro ciclo de crítica y de construcción de universalidad concreta.

Esta dialéctica, en torno a la idea de la verdadera universalización de la cultura occidental, está presente en otros textos.

En América en la historia de 1957, Zea sitúa a América en el horizonte de la historia universal. De acuerdo con su análisis, el mundo grecolatino y cristiano-medieval se escinde en la Época Moderna en una cultura ibera, guardián del pasado, y una cultura occidental, centrada primero en Europa y luego en los Estados Unidos y extendida en nuestros días a todo el planeta, la cual sustituye ese pasado por la búsqueda de bienestar material y libertades democrático-liberales; dando pie a la formación de una América moderna, occidentalizada, la sajona, y marginando de Occidente a un conjunto de pueblos: Iberoamérica, Rusia, la misma Europa con el paso del tiempo. En este marco, Zea argumenta que los nacionalismos antiimperialistas del Siglo XX, "lejos de amenazar a la cultura occidental, le ofrecen las posibilidades de una auténtica universalización” (Zea 1957:108). 
En Dialéctica de la conciencia americana de 1976, ese proceso de expansión occidental sobre el mundo es centrado por Zea en el momento en que los Estados Unidos desplaza a Europa del centro de poder de Occidente, con todo lo que ello ha significado para Latinoamérica. Esta región, al independizarse de España y Portugal, toma como modelos, entre otros pueblos, pero de manera destacada, a los Estados Unidos, pero estos, en vez de aliarse a las fuerzas progresistas de la región, y haciendo prevalecer sus intereses sobre sus valores, una y otra vez se coloca del lado de las fuerzas retrógradas. Los latinoamericanos, al tomar conciencia de esto, exigen que los Estados Unidos antepongan sus valores a sus intereses, y con ello, una auténtica universalización, que en ese caso significa realizar el valor estadounidense de la libertad para todos los hombres y no solo para algunos: "La lucha es ya por la liberación total del hombre" (Zea 1976:353).

En Filosofía de la historia americana de 1978, Zea interpreta la historia de la América ibera a partir de los proyectos que la han impulsado: el proyecto liberador, una de cuyas influencias es la Ilustración, logra la independencia política de la América ibera respecto a España y Portugal, y da origen al proyecto conservador, que pugna por un orden colonial sin las metrópolis, y al proyecto civilizador, que derrota al conservador, pero deja sin resolver problemas como el de la dependencia, manifiesto en su situación subordinada respecto a las mismas naciones modernas cuya guía acepta, haciendo así necesario un renovado proceso de liberación del que forma parte la propuesta de asimilación dialéctica del pasado histórico y que cristaliza en el proyecto asuntivo que Zea ve amanecer con los nacionalismos antiimperialistas en la América Latina del siglo XX. Una asimilación que exige tomar conciencia de la propia historia dependiente y marginal, no como algo distinto de la historia hegemónica del centro, sino como "el otro lado de la moneda del mundo, de nuestro mundo, como totalidad" (Zea 1978:28). Se trata, pues, de transitar de la falsa universalidad que oculta la dominación (la historia eurocéntrica), a una verdadera universalidad (la "conciencia de dependencia"), en la que la dominación, desde la propia realidad, es comprendida como un problema común que reclama una solución igualmente común.

En Discurso desde la marginación y la barbarie de 1988, Zea reflexiona sobre la historia del poder y la dominación en Occidente modernos y contemporáneo y en el mundo clásico y medieval, y lo hace a través del fenómeno clave de la barbarie. Hay barbarie donde sea que unos hombres se consideran a sí mismos y a su cultura (a su logos), como modelo de lo humano y punto de referencia para juzgar la humanidad de los demás hombres y de las otras culturas, pues da pie a que aquellos hombres y culturas que no corresponden al modelo vengan a ser considerados como bárbaros, es decir, como semihumanos o no humanos. En este marco, Zea afirma que la verdadera barbarie no es la de ser diferente a los pueblos poderosos que se ponen a sí mismos como modelo de humanidad, sino la de esos pueblos en la medida que no comprenden que la humanidad de los hombres no radica en ajustarse a un modelo sino en ser diferentes: "El hombre, todo hombre, es igual a cualquier otro hombre. Y esta igualdad no se deriva de que un hombre o un pueblo pueda ser o no copia fiel de otro, sino de su propia peculiaridad. Esto es, un hombre, o un pueblo, es semejante a otros por ser como ellos, distinto, diverso" (Zea 1988a:24).

Como apunta Saladino, humanismo "concreto y universal" (Saladino 2017:52). Ya en América como conciencia Zea caracteriza al ser humano como una "realidad plena de accidentalidades, pero que, por serlo, no podrá modificarla sino accidentalmente, esto es, sin tocar ni alterar su verdadera esencia" (Zea 1953a:87). Esta concepción del hombre como igual en la diferencia constituye un planteamiento más universal que la idea de la verdadera universalización de Occidente, debido a 
que, por una parte, conserva el espíritu de esta última y no limita su alcance a una época histórica, a una cultura o a una región geopolítica y, por otra parte, evita caer una nueva universalidad abstracta, en tanto incorpora la concreción como rasgo fundamental del ser del hombre.

Es pues manifiesta la presencia del universalismo crítico y la dialéctica del universalismo crítico en los diversos momentos y aspectos de la obra de Zea.

\section{Conclusiones}

He interpretado en la obra de Zea el relativismo historicista, el circunstancialismo, el perspectivismo y el enfoque político como principios críticos y la autonomía racional de la teoría y la idea de la universalidad concreta como principios constructivos; y he vinculado la base epistemológica, que comprende esas posturas e ideas, con la base política, a través del enfoque político. Además, mediante la base política, con su problema de la dependencia y su propuesta de asimilación dialéctica, he puesto de relieve el sentido liberador del pensamiento de este filósofo.

A partir de esto, el concepto de universalismo crítico hace explícita la relación entre epistemología y política en esas ideas y posturas; específicamente, entre los procesos teóricos de crítica y universalización operados por el filósofo mexicano y los procesos no teóricos de liberación con los que este filósofo se compromete; y vincula la vertiente crítica de la obra de Zea, que es la más visible, con su vertiente constructiva, conectando así su historicismo y pragmatismo-político con el reconocimiento de la autonomía racional de la teoría y la posibilidad de conocimientos y valores universales.

La dialéctica del universalismo crítico, articulando la base epistemológica y la base política, expone su dinamismo, y muestra cómo las posturas e ideas de Zea pueden ser útiles para el estudio en general de la historia de las ideas y la filosofía de la historia; pero, sobre todo, constituye un camino para estudiar la vida histórica de los conocimientos y valores sin renunciar a la construcción de una auténtica universalidad sobre bases racionales.

Un problema que queda abierto a posterior tratamiento es el de la doble dimensión epistemológica y axiológica de las posturas e ideas aquí estudiadas. Hace falta distinguir la forma en que el universalismo crítico y su dialéctica se despliegan en el ámbito de los conocimientos y en el ámbito de los valores, considerando que cada uno de tales ámbitos posee una racionalidad específica.

Para terminar, recordemos la enseñanza de Zea y reflejémosla sobre su propia obra: al estudiar las ideas del pasado no se trata de copiar los frutos, hay que asumir el espíritu.

\section{Bibliografía}

Beltrán, I. (2017). Dialéctica del reconocimiento. Universalidad y particularidad en la filosofía de la historia de Leopoldo Zea. Thémata, 55, 249-266. https://doi.org/10.12795/themata.2017.i55.11

Beltrán, I. (2020). La dialéctica de la utopía en la primera época de Leopoldo Zea (1940-1954). Utopía y praxis latinoamericana, 25(90), 169-188. https://produccioncientificaluz.org/index.php/utopia/article/view/32357 
Davis, H. (1972). Problemas en la historia de las ideas en Latinoamérica. Latinoamérica, 5, 43-54.

Friz, C. (2019). Revisitando la discusión entre Augusto Salazar Bondy y Leopoldo Zea. La filosofía latinoamericana: el lugar de un diferendo. Revista de Filosofía, 76, 57-74. https://revistafilosofia.uchile.cl/index.php/RDF/article/view/55764/58678

Gómez-Martínez, J. (1997). Leopoldo Zea. Ediciones del Orto.

Gómez-Martínez, J. (2004). Leopoldo Zea: reflexiones para asumir críticamente su obra. Cuadernos Americanos, 5(107), 31-44.

Guadarrama, P. (2013). América en la historia de Leopoldo Zea. En A. Santana. El pensamiento latinoamericano y el centenario de Leopoldo Zea (pp. 35-60). UNAM.

Hale, C. (1970). Sustancia y método en el pensamiento de Leopoldo Zea. Historia Mexicana, 20(2), 285-304. https://historiamexicana.colmex.mx/index.php/RHM/article/view/2510

Hernández, G. (2004). Del "circunstancialismo" de Ortega y Gasset a la "filosofía mexicana" de Leopoldo Zea. UNAM.

Kourím, Z. (1974). Ensayo de la filosofía de la cultura americana: Leopoldo Zea. Latinoamérica, 7, 77-100.

Kourím, Z. (1992). Algunas reflexiones sobre la obra de Leopoldo Zea: los últimos 25 años. En VV.AA. América Latina. Historia y destino (pp. 119-147). UNAM.

Lizcano, F. (2004). Leopoldo Zea. Una filosofía de la historia. UAEMEX.

López, P. (1989). Una filosofía para la libertad. (La filosofía de Leopoldo Zea.) Costa-Amic.

Medin, T. (1983). Leopoldo Zea: ideología, historia y filosofía de América Latina. UNAM.

Medin, T. (2005). Tres senderos y un norte: Leopoldo Zea y la reivindicación de la humanidad latinoamericana. En VV. AA. Leopoldo Zea y la cultura (pp. 39-47). UNAM.

Raat, W. (1970). Ideas e historia en México. Un ensayo sobre metodología. Latinoamérica, 3, 175188.

Reyes-Ruiz, E. (2020). Leopoldo Zea y su discurso filosófico contra la marginalidad (1948-1953). Figuras, 1(3), 8-29. https://revistafiguras.acatlan.unam.mx/index.php/figuras/article/view/114/153

Roig, A. (1981). Filosofía, universidad y filósofos en América Latina. UNAM.

Saladino, A. (2017). Leopoldo Zea. Fundación Ignacio Larramendi.

Shulgovsky, A. (1979). Romanticismo y positivismo en América Latina. Latinoamérica, 12, 27-55.

Vera, M. 1982. La obra filosófica de Leopoldo Zea a la luz de sus críticos. Diánoia, 28(28), 289-307. http://dianoia.filosoficas.unam.mx/index.php/dianoia/article/view/837

Villegas, A. (1960). La filosofía de lo mexicano. FCE.

Villoro, L. (1987). Sobre el problema de la filosofía latinoamericana. Cuadernos americanos, 3(3), 86-104.

Zea, L. (1943). El positivismo en México. El Colegio de México.

Zea, L. (1945). En torno a una filosofía americana. El Colegio de México.

Zea, L. (1948). Ensayos sobre filosofía en la historia. Stylo.

Zea, L. (1949). Dos etapas del pensamiento en Hispanoamérica. Del romanticismo al positivismo. EI Colegio de México.

Zea, L. (1952a). La filosofía como compromiso y otros ensayos. FCE. 
Zea, L. (1952b). Conciencia y posibilidad del mexicano. Porrúa y Obregón.

Zea, L. (1953a). América como conciencia. Cuadernos Americanos.

Zea, L. (1953b). El occidente y la conciencia de México. Porrúa y Obregón.

Zea, L. (1957). América en la historia. FCE.

Zea, L. (1965). El pensamiento latinoamericano. Pomarca.

Zea, L. (1969). La filosofía americana como filosofía sin más. Siglo XXI.

Zea, L. (1974). Dependencia y liberación en la cultura latinoamericana. Joaquín Mortiz.

Zea, L. (1976). Dialéctica de la conciencia americana. Alianza Editorial Mexicana.

Zea, L. (1978). Filosofía de la historia americana. FCE.

Zea, L. (1988a). Discurso desde la marginación y la barbarie. Anthropos.

Zea, L. (1988b). Autobiografía intelectual. Anthropos, 89, 11-21.

Recibido el 17 Sep 2020

Aceptado el 24 Nov 2020 